\title{
ISOMER BEAMS
}

\author{
P.M. WALKER \\ Department of Physics, University of Surrey, \\ Guildford GU2 7XH, UK \\ p.walker@surrey.ac.uk \\ Received (received date) \\ Revised (revised date)
}

\begin{abstract}
As a subset of radioactive beams, isomer beams open up the dimensions of excitation energy and angular momentum. The problem of isomer decay rates is briefly presented, as they are not yet well understood. The use of isomer beams for nuclear structure investigations is reviewed, using examples related to exotic decay modes, charge radii, and Coulomb excitation. There are also unique possibilities for the study of induced electromagnetic depopulation. A key aspect is beam purification, for which different techniques are discussed.
\end{abstract}

\section{Introduction}

Isomers are metastable excited states of atomic nuclei. ${ }^{1,2}$ They can decay by the same mechanisms as unstable ground states, as illustrated in Figure 1. In addition, isomers (but not ground states) can decay electromagnetically, by $\gamma$-ray or conversion-electron emission.

There is no strict lower half-life limit required for an excited nuclear state to be called an isomer. It is rather a practical matter of being able to use the lifetime to distinguish isomer radiations from the majority of "prompt" radiations that are emitted following nuclear reactions. The present work is concerned with isomer beams from electromagnetic separation devices, which typically require about $500 \mathrm{~ns}$ for in-flight separation, and about $50 \mathrm{~ms}$ for isotope separation on-line (ISOL) techniques. A perspective on the range of isomers ${ }^{3}$ with an excitation energy of at least $1 \mathrm{MeV}$ and a half-life exceeding $50 \mathrm{~ms}$, is given in Figure 2. Any of these, as well as others at lower excitation energies, can be considered as potential isomer beams by in-flight or ISOL techniques. A different perspective is given in Figure 3, where the requirement is for $I>10 \hbar$ and $T_{1 / 2}>50 \mathrm{~ms}$.

\section{Added dimensions}

It is evident that isomers give access to the added dimensions of excitation energy and angular momentum, as compared to nuclear ground states. There are essentially three classes of isomer: shape traps, yrast traps, and $K$ traps. It is problematic to 
2 P.M. Walker

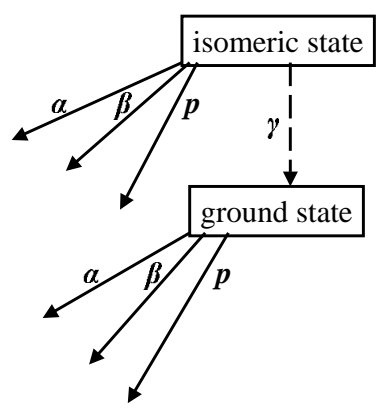

Fig. 1. Isomers can decay by electromagnetic transitions, as well the modes that are available for ground-state decays.

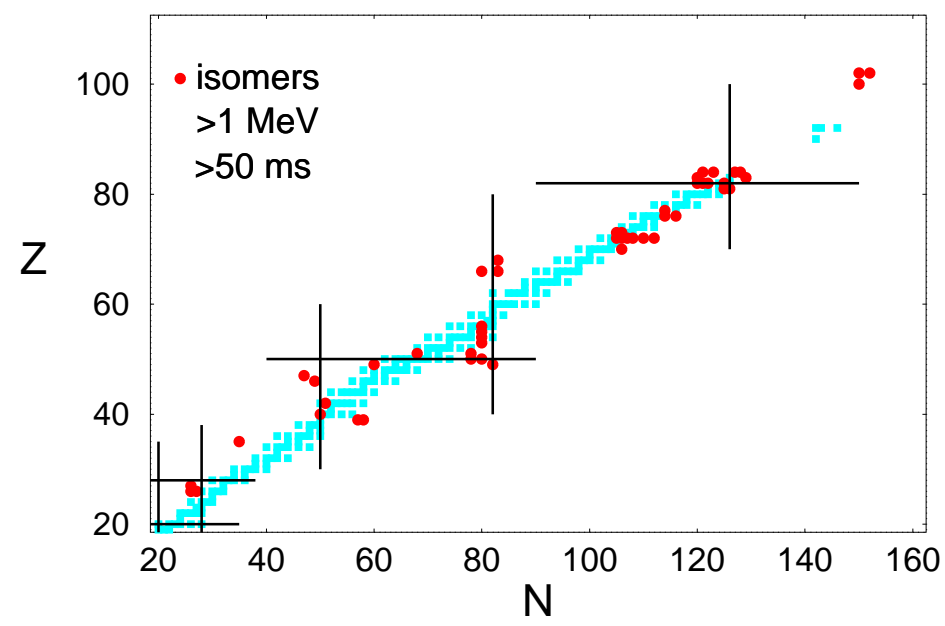

Fig. 2. Illustration of known isomers with $E>1 \mathrm{MeV}$ and $T_{1 / 2}>50 \mathrm{~ms}$, adapted from ref. 1 .

use shape traps as beams, largely due to their shorter half-lives. Interesting though they are, they are not considered further in the present work. Yrast traps are the principal class of isomer, where the isomer is the lowest energy state of a given 


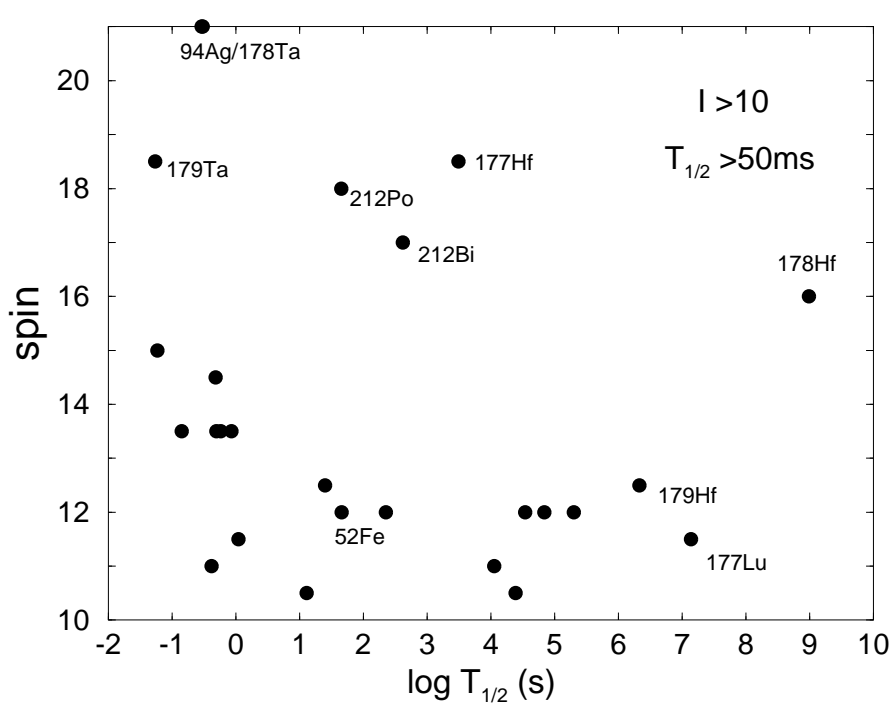

Fig. 3. Illustration of known isomers with $I>10 \hbar$ and $T_{1 / 2}>50 \mathrm{~ms}$, adapted from ref. 4 .

angular momentum in a given nuclide. $K$ traps depend on the orientation of the angular momentum vector, and they occur only in deformed nuclei with an axis of symmetry. They account for most of the examples in Figure 2 that are away from shell closures.

It is expected that additional isomers will be discovered in nuclei far from $\beta$ stability, and various models can be used to make predictions. For example, the neutron-rich nuclide ${ }^{188} \mathrm{Hf}$ is predicted ${ }^{5,4,6}$ to be very favourable for long-lived, high-spin isomers. Indeed, some of the isomers in the ${ }^{188} \mathrm{Hf}$ region are most likely to $\beta$ decay, rather than $\gamma$ decay, and there may be an $A \approx 188$ island of $\beta$-decaying high-spin isomers, only reaching nuclear ground states close to $\beta$ stability. The $A=188$ decay chain is schematically illustrated in Figure 4. One potential complication is that ${ }^{188} \mathrm{Hf}(N=116)$ is itself close to the neutron number $(N=118)$ where a prolate-oblate shape change is expected. ${ }^{7}$ Nevertheless, energy-surface calculations predict well defined prolate, axially symmetric shapes for the ground, $10^{-}$, and $18^{+}$states of ${ }^{188} \mathrm{Hf}$, with $\beta_{2}=0.175,0.177$ and 0.185 respectively, ${ }^{6}$ as illustrated in Figure 5, so that $K$ isomerism is most likely.

Beams of isomers can be used and studied in a variety of inter-related ways: (i) They give access to the structure of exotic nuclei and exotic decay modes at the limits of experimental accessibility. In this category, proton and neutron decays will be considered. (ii) Isomers live long enough to enable static electromagnetic properties to be measured. Here, the charge radii of multi-quasiparticle isomers will be discussed. (iii) Isomer beams can be used to initiate secondary nuclear reactions, and 


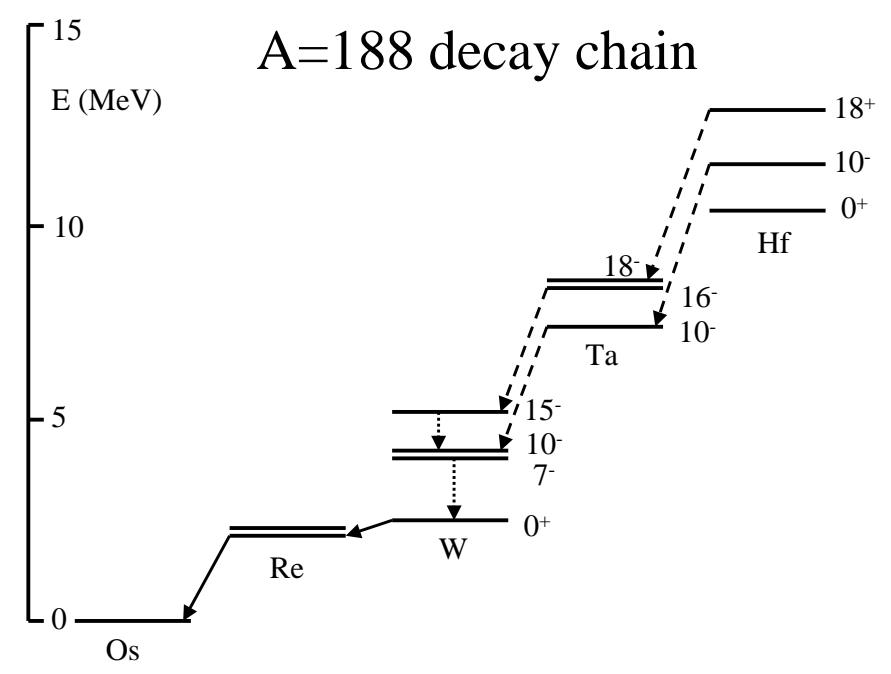

Fig. 4. Illustration of predicted isomers ${ }^{4,5,6}$ and decay modes for the mass- 188 chain.

progress with Coulomb excitation will be presented. An important consideration is always the ability to bring sufficient angular momentum into the initial reaction, which is a critical factor for isomer production by projectile fragmentation ${ }^{8}{ }^{89}$ First, however, consideration is given to isomer decay rates, since these determine their very existence.

\section{Isomer decay rates}

The half-lives of isomers depend on many factors, and so far it is not possible to make reliable predictions, except in special cases. The series of $N=80, I^{\pi}=10^{+}$ seniority isomers in spherical nuclei, for example, show ${ }^{10}$ the effect of the structure of the state to which the isomer decays.

In deformed nuclei, $K$ isomers arise when the decay can only take place by " $K$-forbidden" transitions with $\Delta K>\lambda$, where $\lambda$ is the transition multipolarity. The implied $K$ mixing can be rotational (Coriolis), vibrational ( $\gamma$ tunnelling), or statistical, as discussed in ref. ${ }^{4}$ The statistical, or level-density, effect is illustrated in Figure 6, which has been updated from ref. ${ }^{4}$, including recent data ${ }^{11,12,13,14}$ for ${ }^{183,184} \mathrm{Os},{ }^{174} \mathrm{Yb}$, and ${ }^{174} \mathrm{~W}$, respectively. The curve through the data is based on level-density considerations. ${ }^{15}$ In the graph, the reduced hindrance is defined as $\mathrm{f}_{\nu}=F_{W}^{1 / \nu}$, where $F_{W}$ is the Weisskopf hindrance factor, and $\nu=\Delta K-\lambda$ is the degree of forbiddenness.

The data points that fall below the level-density estimate can be explained, at least in part, by Coriolis $K$ mixing, and especially through the influence of socalled $t$ bands $^{16}$ (the high- $K$ equivalent of $s$ bands). This aspect is illustrated by the two values ${ }^{16}$ given for ${ }^{179} \mathrm{~W}$ : the filled square assumes that the state to which 


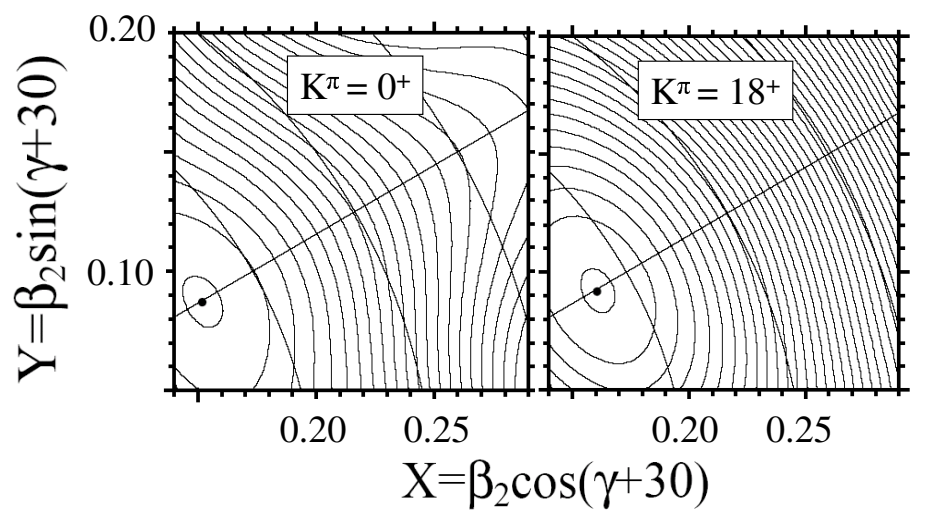

Fig. 5. Configuration-constrained potential energy surfaces ${ }^{6}$ for the ground state and $K^{\pi}=18^{+}$ state in ${ }^{188} \mathrm{Hf}$. Contours are at $200 \mathrm{keV}$ intervals.

the isomer decays has $K=23 / 2$ ( $t$ configuration) while the open square assumes $K=7 / 2$ ( $g$ configuration). The importance of accounting for $t$-configuration mixing is evident, but often this cannot be done quantitatively, and its effect has not been included in evaluating the other data in Figure 6.

The recent data point ${ }^{14}$ for the ${ }^{174} \mathrm{~W}, K^{\pi}=12^{+}$isomer is of special interest because it goes against the $\gamma$-tunnelling prediction. The $6^{+}$isomer decay in ${ }^{174} \mathrm{Yb}$ (not illustrated) is also a problem ${ }^{13}$ for the $\gamma$-tunnelling description. A model is certainly needed that includes both $\gamma$-tunnelling and Coriolis mixing in a consistent framework.

\section{Proton and neutron decays}

The first example of proton radioactivity was found ${ }^{17}$ from a $19 / 2^{-}, 3$-quasiparticle isomer in ${ }^{53} \mathrm{Co}$, with $1.56 \mathrm{MeV}$ proton emission leading directly to the $0^{+}$ground state of ${ }^{52} \mathrm{Fe}$. This kind of nucleon emission from a multi-particle configuration is hard to describe theoretically ${ }^{18,19}$ but leads to the expectation that neutron emission will be a possible mode of radioactive decay on the neutron-rich side of $\beta$ stability. Although the Coulomb barrier would be absent, a substantial centrifugal barrier remains for high-spin-isomer decay. A schematic illustration is given in Figure 7. The requirement might be satisfied in the case of a ${ }^{187} \mathrm{Hf}, 7$-quasiparticle 


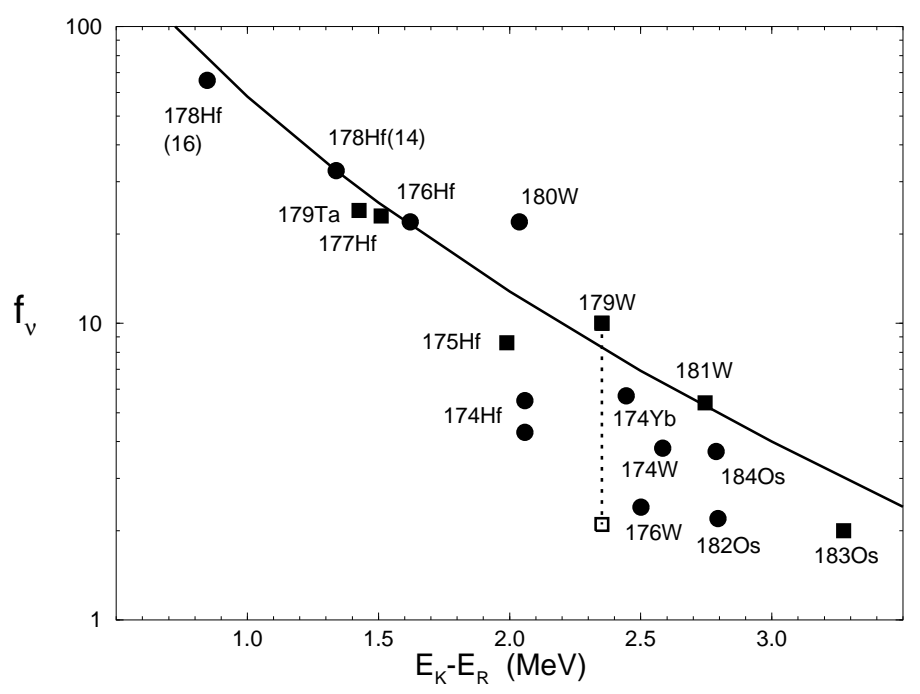

Fig. 6. Reduced-hindrance values for $\Delta K>5, E 2$ and $E 3$ transitions, updated from ref. 4. The circles represent 4-quasiparticle isomers in even-even nuclides, and the squares are for 5quasiparticle isomers in odd-mass nuclides. The data are shown as a function of excitation energy relative to a rigid rotor, with a pairing energy of $0.9 \mathrm{MeV}$ added for odd-mass nuclides. The curve represents a spin-dependent level-density estimate.

isomer ${ }^{20}$ for example. While it would be difficult to observe the associated monoenergetic neutrons, alternative detection methods can be envisaged, such as through the capture of the isomeric nucleus in a storage ring, and the observation of the product nucleus when the isomer decays. Such single-ion decay observations have been clearly identified for other decay modes ${ }^{21}$ in the ESR at GSI.

Multi-quasiparticle proton-emitting isomers have also been found ${ }^{9,22}$ in ${ }^{54} \mathrm{Ni}$ and ${ }^{94} \mathrm{Ag}$. Furthermore, 2-proton emission has been identified from the ${ }^{94} \mathrm{Ag}$ isomer. ${ }^{23}$ It seems that the continuing exploration of isomers close to the drip lines will most likely reveal further examples of exotic decay modes, with important physics implications.

\section{Charge radii}

A very productive use of low-energy ion and atom beams has been for the investigation of nuclear charge radii and electromagnetic moments, by collinear laser spectroscopy. ${ }^{24}$ The method is well suited to isomers with $T_{1 / 2}>50 \mathrm{~ms}$, and refractory elements such as hafnium ${ }^{25}$ can be studied with ion-guide techniques. It is particularly intriguing that isomer charge radii are found ${ }^{26,27}$ to be smaller than their corresponding ground-state radii. This seems to be related to the blocking 

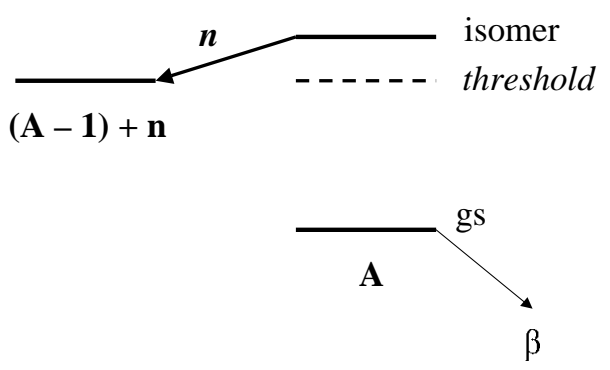

Fig. 7. Schematic illustration of neutron decay from an isomer above the neutron separation energy. Such decay has not yet been observed.

of pairing correlations by excited quasiparticles, with a consequent reduction in surface diffuseness, but a quantitative understanding has not yet been found.

\section{Coulomb excitation}

With the on-going development of radioactive-beam facilities, which generate intense beams of unstable nuclides at energies close to and above the Coulomb barrier, there is the possibility to investigate nuclear reactions with isomer beams. The Coulomb excitation of isomeric states is one of the most straightforward isomer interactions to study, and some results are already available.

Pioneering work, with inverse fusion-evaporation reactions to generate the isomer beam, was carried out at the HSIB facility at RIKEN, where secondary Coulomb excitation of the $2.4 \mu \mathrm{s}, 8^{-}$isomer in ${ }^{174} \mathrm{Hf}$ was successfully observed..$^{28}$

More recently ${ }^{29}$ secondary Coulomb excitation of the $4 \mathrm{~m}, 66^{-}$isomer in ${ }^{68} \mathrm{Cu}$ has been studied at CERN-ISOLDE following proton spallation reactions. Here, attention is drawn to two remarkable features of this study. First, laser resonance ionisation was used to generate an almost pure, accelerated isomer beam - a landmark achievement. Second, the observed Coulomb excitation is associated with isomer depopulation, i.e. at least some of the decay of the Coulomb-excited states bypasses the isomer and proceeds promptly to the ground state. This is of general interest $^{2,30}$ with regard to the quest to control, and indeed enhance, isomer decay rates by electromagnetic processes (which have no influence on ground-state decay rates). Purified isomer beams present a new and unique opportunity to study induced electromagnetic depopulation. 


\section{Isomer beam purification}

As illustrated above, isomer beams present a range of openings for nuclear structure investigations, from $\mathrm{keV}$ energies upwards, and high-energy isomer beams offer novel options specifically for secondary nuclear reactions. However, beam purification is a key issue. In general, mass separators have insufficient resolution to distinguish isomers from ground states.

So far, there appear to be three distinct ways to achieve isomer-beam purification. The simplest, conceptually, is to choose an isomer which is longer lived than its respective ground state, and to wait for the ground state to decay. This is a very restrictive condition, but may be used with, for example, the $45 \mathrm{~s}, 18^{+}$isomer of ${ }^{212} \mathrm{Po}$, where the ground-state half-life is just $300 \mathrm{~ns}$.

Secondly, the method of laser resonance ionisation can be highly selective, as discussed above; and thirdly, storage rings ${ }^{21,31}$ offer sufficient resolution, at least in principle, to physically remove a ground state and leave its isomer.

In addition, for $\mu$ s isomers produced initially in-flight by fusion-evaporation or projectile-fragmentation reactions, it is possibile ${ }^{28}$ to use time correlations with the isomer decay radiations to select events associated with secondary Coulomb or inelastic excitations. More complex possibilities of this type, i.e. exploiting timecorrelated decays, include secondary transfer reactions to $\mu$ s isomers.

\section{Outlook}

The opportunities with isomer beams are many and varied, and only the first steps have so far been taken to exploit them. The future utility of such beams depends on the combination of appropriate beam energy, beam intensity, beam purity, and other aspects of beam quality - spatial, energy and time resolutions. While some of these requirements are integrated into on-going radioactive-beam developments, other isomer-specific features will need separate attention; and there are many open questions, such as whether it will be possible to identify neutron radioactivity from isomers in neutron-rich nuclides.

\section{Acknowledgements}

This work has been supported by the UK Engineering and Physical Sciences Research Council. Special thanks for stimulating discussions, and access to unpublished data, go to Paul Campbell, Jeff Carroll, Georgi Georgiev, Yuri Litvinov, Zsolt Podolyák and Furong Xu.

\section{References}

1. P. M. Walker and G. D. Dracoulis, Nature 399 (1999) 35.

2. P. M. Walker and J. J. Carroll, Physics Today 58-6 (2005) 39.

3. G. Audi, O. Bersillon, J. Blachot and A. H. Wapstra, Nucl. Phys. A729 (2003) 3.

4. P. M. Walker and G. D. Dracoulis, Hyp. Int. 135 (2001) 83. 
5. K. Jain et al., Nucl. Phys. A591 (1995) 61.

6. F. R. Xu, private communication.

7. P. M. Walker and F. R. Xu, Phys. Lett. B635 (2006) 286.

8. Z. Podolyák et al., Phys. Lett. B632 (2006) 203,

9. Z. Podolyák, this conference.

10. J. J. Valiente-Dobon et al., Phys. Rev. C69 (2004) 024316.

11. T. Shizuma et al., Nucl. Phys. $\mathbf{A 6 9 6}$ (2001) 337.

12. T. Shizuma et al., Phys. Rev. C65 (2002) 064310.

13. G. D. Dracoulis et al., Phys. Rev. C71 (2005) 044326; C73 (2006) 019901.

14. S. K. Tandel et al., Phys. Rev. C73 (2006) 044306.

15. P. M. Walker et al., Phys. Lett. B408 (1997) 42.

16. P. M. Walker et al., Nucl. Phys. A568 (1994) 397.

17. K. P. Jackson et al., Phys. Lett. B33 (1970) 281.

18. L. K. Peker, E. I. Volmyansky, V. E. Bunakov and S. G. Ogloblin, Phys. Lett. B36 (1971) 547.

19. V. P. Bugrov et al., Sov. J. Nucl. Phys. 42 (1985) 34.

20. P.M. Walker, AIP Conf. Proc. 819 (2006) 16.

21. Y. A. Litvinov et al., Nucl. Phys. A756 (2005) 3.

22. I. Mukha et al., Phys. Rev. Lett. 95 (2005) 022501.

23. I. Mukha et al., Nature 439 (2006) 298.

24. J. Billowes and P. Campbell, J. Phys. G21 (1995) 707.

25. J. M. G. Levins et al., Phys. Rev. Lett. 82 (1999) 2476.

26. N. Boos et al., Phys. Rev. Lett. 72 (1994) 2689

27. P. Campbell, private communication.

28. T. Morikawa et al., Phys. Lett. B350 (1995) 169.

29. G. Georgiev, CERN Courier, Dec. 2005, and this conference.

30. J. J. Carroll, this conference.

31. F. Bosch et al., Int. J. Mass Spectrometry 251 (2006) 212. 